\title{
Change in Intrathoracic Pressure in Rats with Spontaneous and Controlled Ventilation during Microgravity by Parabolic Flight
}

\author{
Taro Miyahara GOTOH, Nobuhiro FUJIKI, Kunihiko TANAKA, and Hironobu MORITA \\ Department of Physiology, Gifu University School of Medicine, Gifu, 501-1194 Japan
}

\begin{abstract}
We previously reported that the intrathoracic pressure (ITP) decreases and the transmural pressure of the aortic wall (TMP) increases during $4.5 \mathrm{~s}$ of microgravity $(\mu \mathrm{G})$ induced by free drop. To examine the ITP response to a longer period of $\mu \mathrm{G}$ in the absence of the respiratory rate (RR) decrease, i.e., bradypnea, which occurs at the onset of $\mu \mathrm{G}$, we measured the aortic blood pressure at the diaphragma level (AP) and ITP. We then calculated the TMP at the aortic arch level during $20 \mathrm{~s}$ of $\mu \mathrm{G}$ induced by parabolic flight in anesthetized rats $(n=7)$ with either spontaneous ventilation (SPN-V) or controlled ventilation (CONT-V). In the SPN-V group, the bradypnea was observed in all rats after the onset of the
\end{abstract}

$\mu G$ (RR change $-13.9 \pm 2.9 / \mathrm{min})$. The ITP during $\mu \mathrm{G}(-9.3 \pm 0.9 \mathrm{mmHg})$ was significantly lower than that during $1 \mathrm{G}(-7.7 \pm 0.9 \mathrm{mmHg})$, and the TMP was significantly increased during $\mu \mathrm{G}$ (112 $\pm 6 \mathrm{mmHg})$ compared to $1 \mathrm{G}(103 \pm 5 \mathrm{mmHg})$. Similar changes in ITP and TMP were observed in the CONT-V group: During $\mu \mathrm{G}$ and $1 \mathrm{G}$, respectively, the ITP was $-8.4 \pm 0.6 \mathrm{mmHg}$ and $-5.9 \pm$ $0.7 \mathrm{mmHg}$, and the TMP was $112 \pm 6 \mathrm{mmHg}$ and $101 \pm 6 \mathrm{mmHg}$, whereas no change in RR was observed because of the controlled ventilation. These results show that the ITP decreases and the TMP increases during $\mu \mathrm{G}$, and they are not affected by a disturbance of respiratory rhythm. [The Japanese Journal of Physiology 55: 69-74, 2005]

Key words: intrapleural pressure, space, arterial blood pressure, transmural pressure, fluid shift.

$\mathrm{T}_{\mathrm{s}}$ on the cardiovascular system. Astronauts experience facial edema, nasal congestion, and negative water balance during space flight and orthostatic intolerance after returning to earth [1]. A cephalad fluid shift resulting from the disappearance of the gravitational pressure gradient (or hydrostatic pressure gradient) along the head-to-foot axis and the adaptive responses to it are thought to be major causes of these harmful symptoms [2]. Furthermore, we previously reported that the decrease in intrathoracic pressure (ITP) also plays an important role in the cardiovascular responses in anesthetized rats during $4.5 \mathrm{~s}$ of $\mu \mathrm{G}$ induced by free drop $[3,4]$. However, two points arise concerning our previous results. The first is that the ITP decrease may be due to a change in the respiratory rhythm, and some of our previous ITP recordings did show the bradypnea just after the onset of $\mu \mathrm{G}$; furthermore, in parabolic flight experiments, other researchers have also noted a change in respiratory rhythm just after an onset of the $\mu \mathrm{G}$ [5]. Generally, the minute ventilation is the product of the respiratory rate (RR) and the tidal volume and is maintained at a certain level by various mechanisms [6]. When a decrease in RR is induced, a more intensive contraction of the respiratory muscles during inspiration is necessary to keep the minute ventilation, which may result in the decrease in ITP. The second point is the duration of the ITP decrease. In our previous experiment, the duration of the $\mu \mathrm{G}$ was as short as $4.5 \mathrm{~s}$, thus it cannot be denied that the ITP change is only transient. White et al. [7] hypothesized on theoretical grounds that $\mu \mathrm{G}$ removes the weight of the chest wall and causes a relaxation of the chest, thus increasing the closed chest cavity and resulting in the ITP decrease. It is required that the decrease in ITP continues longer for this hypothesis to be the truth. 
The aims of the present study were therefore twofold. The first was to examine whether the ITP decrease occurred in the absence of any disturbance of respiratory rhythm. The second was to examine whether it was seen during a longer period of $\mu \mathrm{G}$. To do so, we measured the aortic blood pressure (AP) and ITP, then calculated the transmural pressure of the aortic wall (TMP) in anesthetized rats with either spontaneous ventilation $(\mathrm{SPN}-\mathrm{V})$ or controlled ventilation (CONT$\mathrm{V})$ during $20 \mathrm{~s}$ of $\mu \mathrm{G}$ induced by parabolic flight.

\section{Methods}

Male Sprague-Dawley rats weighing $347 \pm 6 \mathrm{~g}$ ( $n=$ 7) were used. The animals were maintained in accordance with the "Guiding Principles for Care and Use of Animals in the Field of Physiological Science" of the Physiological Society of Japan. The experimental protocol was approved by the Animal Research Committee of Gifu University.

On the experimental day, the rats were anesthetized with urethane and $\alpha$-chloralose (500 and $50 \mathrm{mg} / \mathrm{kg}$ body wt, respectively, ip); a polyethylene catheter (PE50, Becton Dickinson, Sparks, USA) for AP measurement was then inserted into the abdominal aorta at a level immediately below the diaphragma through the femoral artery, and another catheter for drug administration was inserted into the inferior vena cava through the femoral vein. Both catheters were exteriorized at the back of the neck. A tracheal cannula was inserted for controlled ventilation and the tip of a telemetry transmitter probe (TA11PA-C40, Data Science International, St. Paul, USA) for ITP measurement was inserted into the intrapleural space through the fifth intercostal space. After surgery, the rats were fixed in a $30^{\circ}$ head-up position in order to observe the responses induced by the disappearance of the gravitational pressure gradient in the head-to-foot axis caused by $\mu \mathrm{G}[8,9]$. The AP catheter was connected to a pressure transducer (MP5200, Baxter, Deerfield, USA), which was fixed at the same level as the catheter tip to avoid a gravitational pressure difference between the two [10]. The signal from the AP transducer was transmitted to an amplifier (AP-621G, Nihon Kohden, Tokyo, Japan). The ITP signal was sent to a PhysioTel Receiver (RLA1020, Data Science International, St. Paul, USA) and the output relayed through a calibrated pressure output adapter (R11CPA, Data Science International, St. Paul, USA) and dual ambient pressure monitor (C11PR, Data Science International, St. Paul, USA). These biological signals and $G$ level data (measured by a vertical accelerometer) were stored in a DAT data recorder (RD-145T, TEAC, Tokyo, Japan).
The parabolic flight experiments were carried out at Diamond Air Service (Nishikasugai-Gun, Aichi 480-0202, Japan; http://www.das.co.jp). The parabolic maneuvers were performed in a specially modified Gulfstream-2 jet aircraft (G-1159, Grumman American Aviation, USA). A parabola consisted of three phases: control $1 \mathrm{G}$, hypergravity of about $1.8 \mathrm{G}$, and $\mu \mathrm{G}$. During each $1 \mathrm{~h}$ flight, 10 parabolas were performed, with a 4-6 min interval between two consecutive parabolas. During the first and last 5 parabolas, data were collected from the same rats with SPN-V or CONT-V, respectively. CONT-V was performed by connecting the tracheal cannula to a mechanical ventilator (SAR-830/P, IITC, Woodland Hills, USA) operating in volume control mode, and the tidal volume and respiratory rate were set at $10 \mathrm{ml} / \mathrm{kg}$ and $60 / \mathrm{min}$, respectively. To eliminate SPN-V during CONT-V, a muscle relaxant drug (pancuronium bromide, $1 \mathrm{mg} / \mathrm{kg}$ body wt, iv) was administered.

At the end of the experiment, the data were played back and sampled by the use of an analog-digital converter (PowerLab, ADinstruments, Castle Hill, Australia) at a rate of 100 samples/s. The RR was calculated by using the ITP measurements. The TMP at the aortic arch level, where the baroreceptor exist, was calculated.

$\mathrm{AP}_{\text {arch }}=\mathrm{AP}-\mathrm{GD} \times \mathrm{Gz}$

$\mathrm{TMP}=\mathrm{AP}_{\text {arch }}-\mathrm{ITP}$

where $\mathrm{AP}_{\text {arch }}$ is $\mathrm{AP}$ at the aortic arch level, GD is the gravitational pressure difference between the catheter tip and the aortic arch, and Gz is the gravity.

All variables for each rat were averaged over five parabolas and the averaged data used as a single data point for that rat. For statistical analysis, the AP, ITP, and TMP were averaged over $10 \mathrm{~s}$ during $1 \mathrm{G}$ and over $10 \mathrm{~s}$ during the second half of $\mu \mathrm{G}$. The results were compared by two-way analysis of variance (ANOVA) with gravity and respiratory method (i.e. SPN-V or CONT-V) as factors. The significance level was set at $p<0.05$.

\section{Results}

Figure 1 shows typical recordings of the gravity, AP, ITP, and RR responses to parabolic flight. The upper panel shows the responses during SPN-V. In this case, the AP decreased slightly during hypergravity, then increased during $\mu \mathrm{G}$. The ITP slightly increased during hypergravity and decreased during $\mu \mathrm{G}$; as mentioned in the introduction, bradypnea was observed at the onset of $\mu \mathrm{G}$ (arrow). The lower panel shows the responses during CONT-V. The AP did not change during hypergravity, and it increased during 
$\mu \mathrm{G}$. The ITP slightly increased during hypergravity and decreased during $\mu \mathrm{G}$; since the respiratory rhythm was controlled by the mechanical ventilator, no disturbance in respiratory rhythm was observed throughout the recording. Figure 2 shows the averaged data for the seven rats. The averaged gravity level during $\mu \mathrm{G}$ was $0.01 \pm 0.00 \mathrm{G}$. The AP gradually decreased during hypergravity and started to increase just after the onset of $\mu \mathrm{G}$ in both the SPN-V and CONT-V groups. The ITP was higher during $1 \mathrm{G}$ in the CONT-V group (-5.9 $\pm 0.7 \mathrm{mmHg})$ than in the SPN-V group $(-7.7 \pm 0.9$ $\mathrm{mmHg}$ ) because of the positive pressure ventilation. It then increased slightly during hypergravity and decreased suddenly during $\mu \mathrm{G}$ in both groups. Because of the additive effect of the changes in AP and ITP, the changes in the TMP were more marked than those in

\section{A: Spontaneous Ventilation}

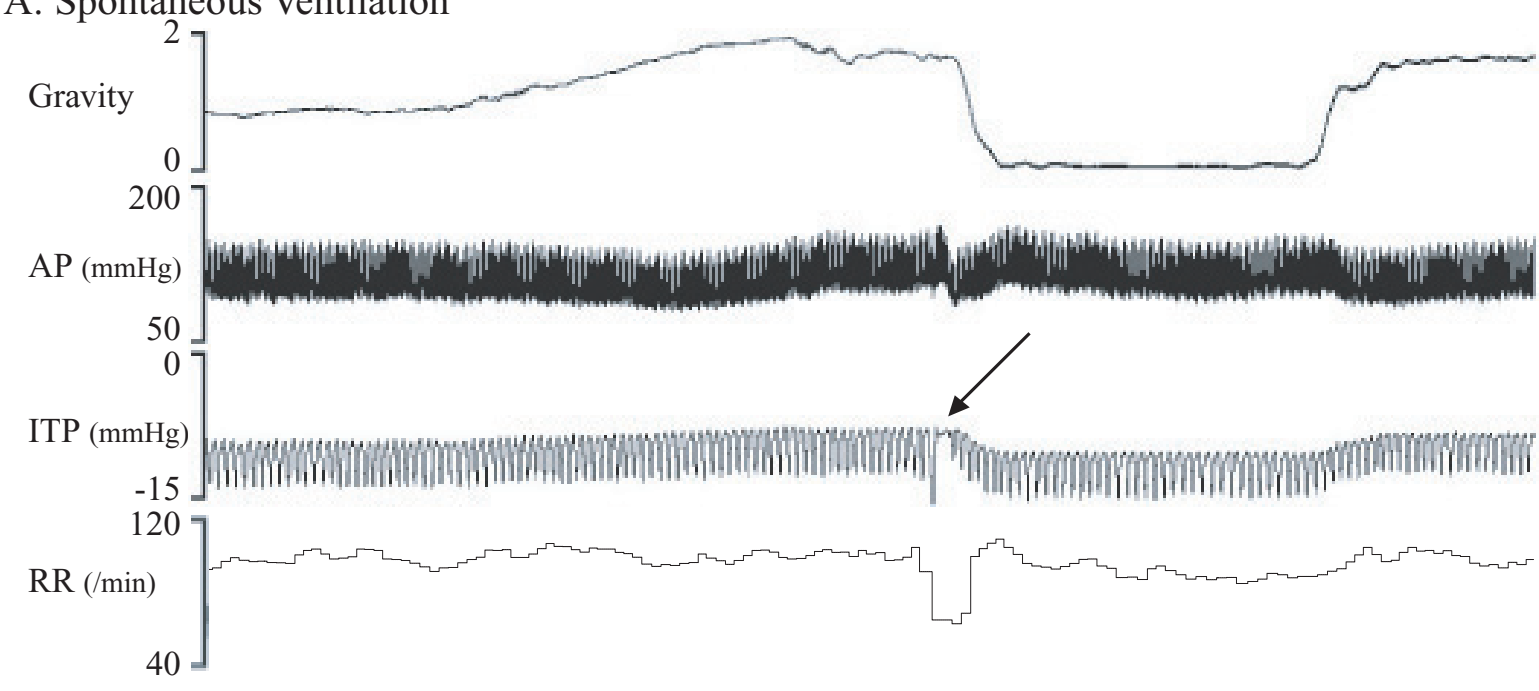

B: Controlled Ventilation

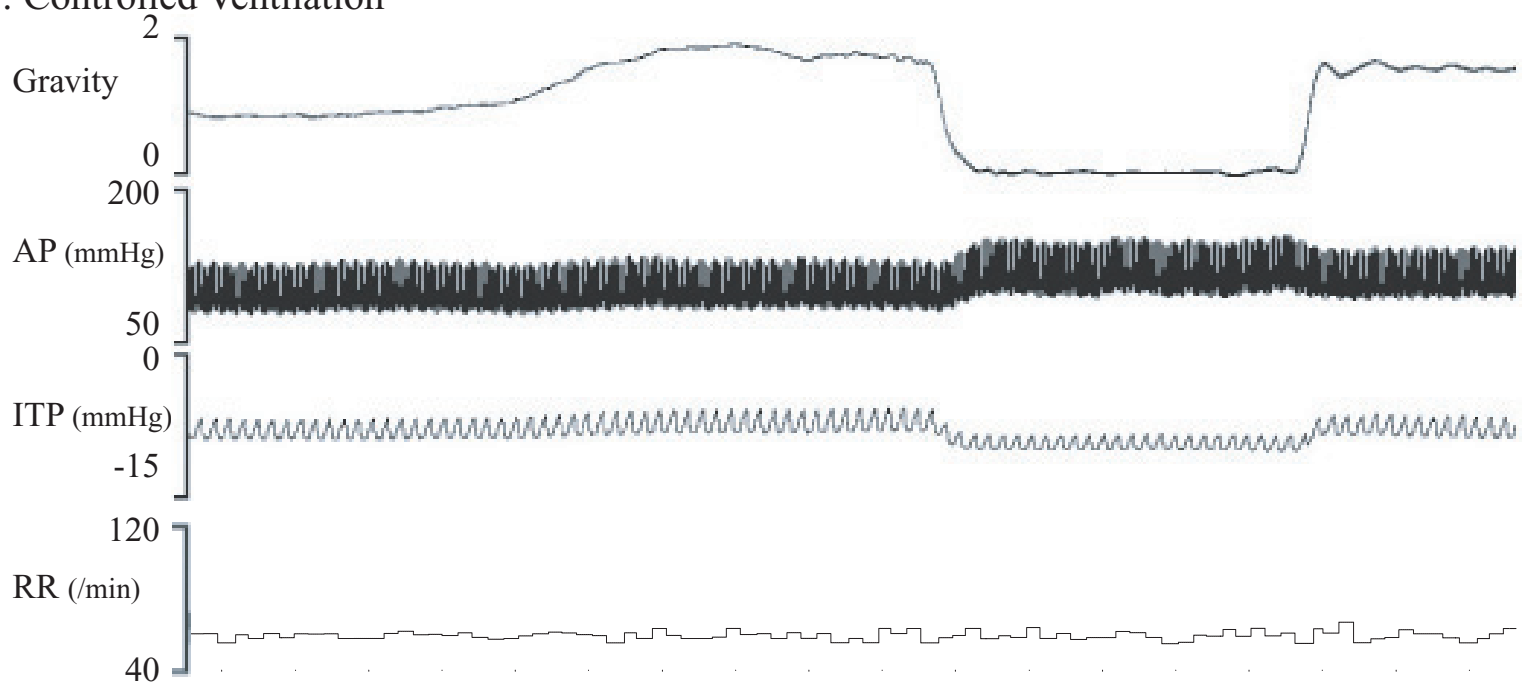

$10 \mathrm{sec}$

Fig. 1. Original recordings showing changes in gravity, aortic blood pressure (AP), and intrathoracic pressure (ITP) and respiratory rate (RR) during parabolic flight in an anesthetized rat with spontaneous ventilation $(A)$ or controlled ventilation (B). A disturbance of the respiratory rhythm was observed just after the onset of $\mu \mathrm{G}$ during spontaneous ventilation (arrow). 
the AP. The RR during control $1 \mathrm{G}$ was $84.1 \pm 5.0$ and $58.9 \pm 0.0 / \mathrm{min}$ for the SPN-V and CONT-V groups, respectively. The bradypnea was observed after the onset of the $\mu \mathrm{G}$ in all seven rats of the SPN-V group, but in no rat of the CONT-V group. Since the time delay of the bradypnea after an onset of the $\mu \mathrm{G}$ differed among rats from 0 to $5 \mathrm{~s}$, and since its duration was short, the averaged drop in the RR was small in Fig. 2; however, the maximum RR drop averaged over seven rats was $-13.9 \pm 2.9 / \mathrm{min}(p=0.002$ vs. $1 \mathrm{G}$ control) . On the other hand, no change in RR was observed in CONT-V group as expected. A statistical analysis

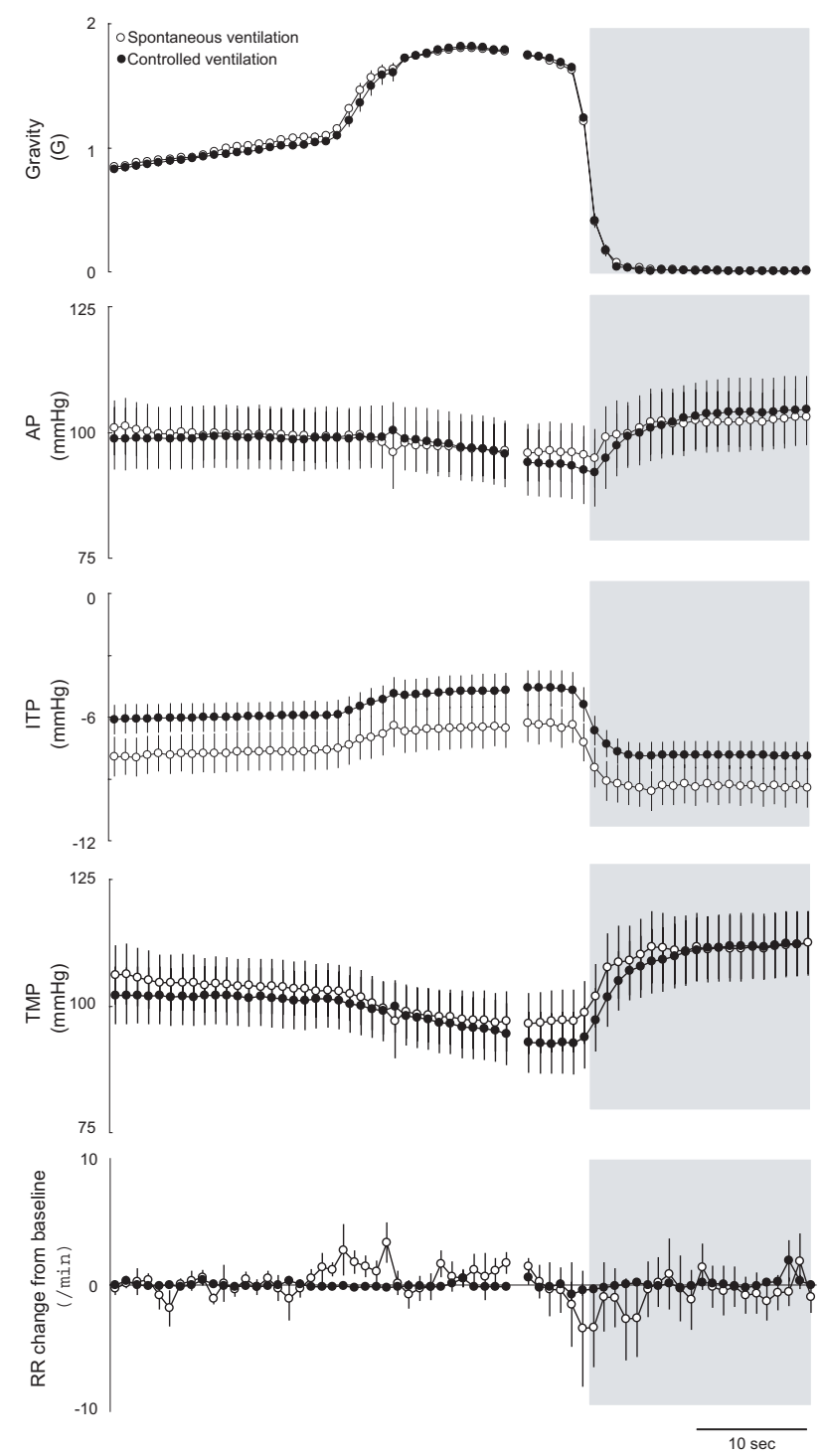

Fig. 2. Averaged data for gravity, aortic blood pressure (AP), intrathoracic pressure (ITP), transmural pressure of the aortic wall (TMP), and respiratory rate (RR) change in anesthetized rats $(n=7)$ with spontaneous ventilation (open circles) or controlled ventilation (solid circles). The horizontal bar shows a $10 \mathrm{~s}$ interval, and the gray area shows the period of $\mu \mathrm{G}$. showed a significant change in the AP, ITP, and TMP during $\mu \mathrm{G}$ compared to during $1 \mathrm{G}$ in both the SPN-V and CONT-V groups (Fig. 3).

\section{Discussion}

The present results clearly showed that during $\mu \mathrm{G}$ the ITP decreased and the TMP increased, and that these changes occurred independent of any disturbance of the respiratory rhythm, since they were seen in both the SPN-V and CONT-V groups.

Although we have previously used the AP and ITP to calculate the TMP in a free-drop study, the duration of $\mu \mathrm{G}$ was only $4.5 \mathrm{~s}$, so only initial responses could be measured $[3,4]$. In the present parabolic flight study, we showed for the first time that the ITP decrease was maintained throughout $20 \mathrm{~s}$ of $\mu \mathrm{G}$. One defect of parabolic flight is that $\mu \mathrm{G}$ is usually preceded by hypergravity of $1.8 \mathrm{G}$, which complicates the interpretation of the results because the results during $\mu \mathrm{G}$ might be affected by the preceding hypergravity. However, the combined results for the free drop and parabolic flight studies provide strong evidence that the ITP decreases during $\mu \mathrm{G}$.

The ITP change is important because of its effects on the circulatory system. Several conflicting effects on the circulatory system have been reported during $\mu \mathrm{G}$. For example, it is known that $\mu \mathrm{G}$ elicits a cephalad fluid shift and causes an increase in venous return, shown by an increase in left atrial diameter, left ventricular end-diastolic volume, and cardiac output [1113], suggesting that central venous pressure, thought to be an indicator of cardiac filling pressure, should be increased; however, it shows a significant decrease $[11,14]$. Furthermore, during the initial phase of $\mu \mathrm{G}$, aortic nerve activity is increased, showing that the baroreceptor was stimulated and suggesting that the aortic pressure was increased; however, aortic pressure is not affected [4]. These apparent discrepancies result from the inappropriate use of the intravascular pressure or aortic pressure to measure vascular filling under conditions of $\mu \mathrm{G}$ instead of the TMP, calculated by subtracting the extravascular pressure (i.e., ITP) from the intravascular pressure (i.e., AP or CVP).

The detailed mechanisms of the disturbance of the respiratory rhythm that occurs at the onset of $\mu \mathrm{G}$ are unclear. One possible explanation is an interaction between the cardiovascular and respiratory control systems. It is well documented that a wide variety of sensory inputs from the cardiovascular and respiratory organs, including those from arterial baroreceptors, arterial chemoreceptors, and mechano- and chemoreceptors in the respiratory wall, terminate in 
ITP Change during $\mu \mathrm{G}$
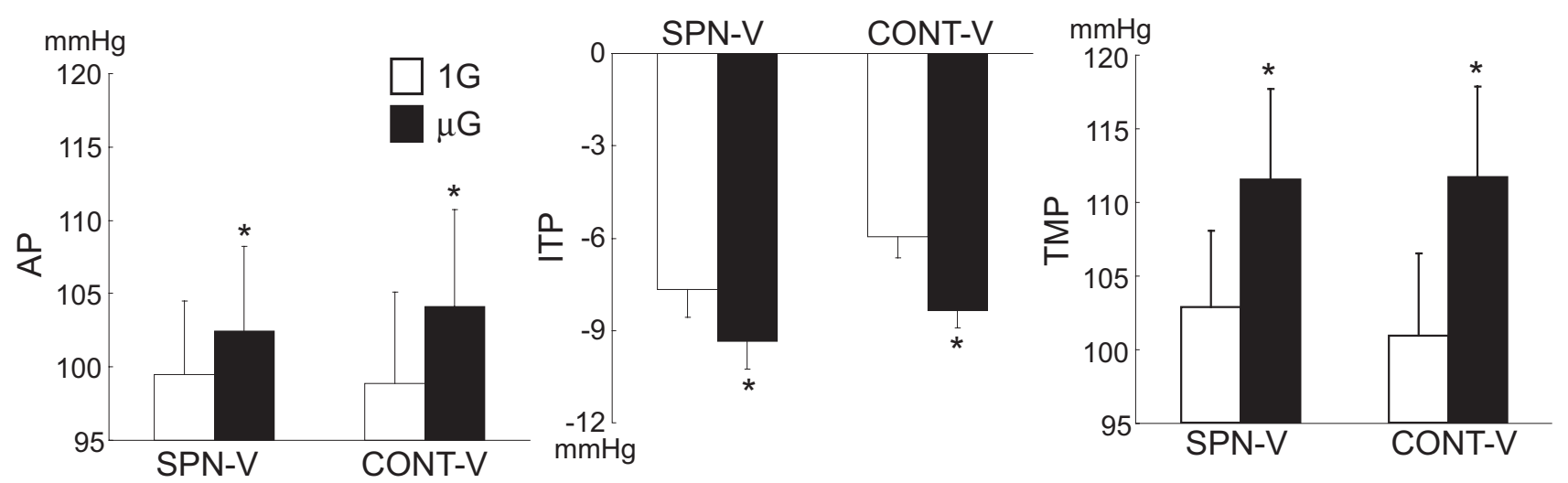

Fig. 3. Aortic blood pressure (AP), intrathoracic pressure (ITP), and transmural pressure of the aortic wall (TMP) in the spontaneous ventilation (SPN-V) group and the controlled ventilation (CONT-V) group. The values shown are the mean \pm standard error. ${ }^{*} p<0.05$, significantly different from the $1 \mathrm{G}$ control value.

the nucleus of the solitary tract and that these inputs show a large overlap $[15,16]$. It has been reported that baroreceptor stimulation, by decreasing the carotid sinus pressure, causes a decrease in respiratory frequency [17]. As mentioned above, both our previous and present results showed that the baroreceptor was stimulated during $\mu \mathrm{G}$, and this may cause the change in respiratory rhythm.

To estimate the TMP at the thoracic region, two essential variables are AP at the thoracic region and ITP; however, we measured AP at the diaphragma level in the present study. Whereas the postural change from standing to supine induces an intravascular pressure increase at the cranial region and a decrease at the lower extremities, there should be an intermediate point where the intravascular pressure remains constant. This point is called the hydrostatic indifferent point, which exists around the diaphragma [1]. By measuring AP at this point, we can estimate the dynamic component of the aortic pressure, which is generated by the heart pumping, without static component, which is generated by the gravity. Although we measured AP at this hydrostatic indifferent point, the AP at the thoracic region can be calculated by subtracting the gravitational pressure difference between the hydrostatic indifferent point and the aortic arch.

Although it was demonstrated that the ITP decrease occurred independently of the respiratory rhythm in the present study, one confusing point for the data interpretation is that the respiratory mode also differed between the SPN-V and CONT-V groups, i.e., negative pressure ventilation and positive pressure ventilation for the SPN-V and CONT-V groups, respectively. Further study is necessary to reveal the relationship between the respiratory system and the ITP during $\mu \mathrm{G}$.
In conclusion, acute $\mu \mathrm{G}$ causes a decrease in the ITP and an increase in the TMP. These changes occur independently of a disturbance in respiratory rhythm. This ITP decrease is one of the key factors inducing cardiovascular responses during $\mu \mathrm{G}$.

We are grateful to the staff members of the Diamond Air Service. This study was supported by a grant-in-aid for Scientific Research from the Ministry of Education, Culture, Sports, Science and Technology of Japan, and a "Ground Research for Space Utilization" research grant from the National Space Development Agency of Japan and the Japan Space Forum.

\section{REFERENCES}

1. Blomqvist CG and Stone HL: Cardiovascular adjustments to gravitational stress. In: Handbook of Physiology, The Cardiovascular System III, ed. Shepherd JT, Abboud FM, and Geiger SR, American Physiological Society, Bethesda, pp 1025-1063, 1983

2. Levy MN and Talbot JM: Cardiovascular deconditioning of space flight. Physiologist 26: 297-303, 1983

3. Morita H, Fujiki N, Gotoh T, Matsuda T, Shuang G, and Tanaka K: Relationship between transmural pressure and aortic diameter during free drop-induced microgravity in anesthetized rats. Jpn J Physiol 53: 151-155, 2003

4. Morita H, Tsuchiya Y, Miyahara T, Tanaka K, and Fujiki $\mathrm{N}$ : Acute response of aortic nerve activity to free dropinduced microgravity in anesthetized rats. Neurosci Lett 304: 33-36, 2001

5. Iwase S, Mano T, Cui J, Kitazawa H, Kamiya A, Miyazaki S, Sugiyama Y, Mukai C, Kohno M, and Nagaoka S: Changes in muscle sympathetic nerve activity and effect of breathing maneuvers during microgravity induced by parabolic flight in humans. Environ Med 42: 152-155, 1998

6. Guyton CA: Regulation of respiration. In: Textbook of Medical Physiology, Saunders, Philadelphia: pp 444-453, 1991 


\section{T.M. GOTOH et al.}

7. White RJ and Blomqvist CG: Central venous pressure and cardiac function during spaceflight. J Appl Physiol 85: 738-746, 1998

8. Gotoh TM, Fujiki N, Tanaka K, Matsuda T, Gao S, and Morita $\mathrm{H}$ : Acute hemodynamic responses in the head during microgravity induced by free drop in anesthetized rats. Am J Physiol Regul Integr Comp Physiol 286 R1063-R1068, 2004

9. Gotoh TM, Fujiki N, Matsuda T, Gao S, and Morita H: Cerebral circulation during acute microgravity induced by free drop in anesthetized rats. Jpn J Physiol 53: 223-228, 2003

10. Gotoh TM, Fujiki N, Matsuda T, Gao S, and Morita H: Roles of baroreflex and vestibulosympathetic reflex in controlling arterial blood pressure during gravitational stress in conscious rats. Am J Physiol Regul Integr Comp Physiol 286: R25-R30, 2004

11. Buckey Jr JC, Gaffney FA, Lane LD, Levine BD, Watenpaugh DE, Wright SJ, Yancy CW Jr, Meyer DM, and Blomqvist CG: Central venous pressure in space. J Appl Physiol 81: 19-25, 1996

12. Johns JP, Vernalis MN, Karemaker JM, and Latham RD:
Doppler evaluation of cardiac filling and ejection properties in humans during parabolic flight. J Appl Physiol 76: 2621-2626, 1994

13. Videbaek $R$ and Norsk P: Atrial distension in humans during microgravity induced by parabolic flights. J Appl Physiol 83: 1862-1866, 1997

14. Foldager N, Andersen TA, Jessen FB, Ellegaard P, Stadeager $C$, Videbaek $R$, and Norsk P: Central venous pressure in humans during microgravity. J Appl Physiol 81: 408-412, 1996

15. Taylor EW, Jordan D, and Coote JH: Central control of the cardiovascular and respiratory systems and their interactions in vertebrates. Physiol Rev 79: 855-916, 1999

16. Coleridge $\mathrm{MH}$, Coleridge $\mathrm{CJ}$, and Jordan D: Integration of ventilatory and cardiovascular control systems. In: The Lung: Scientific Foundation, 2nd ed, LippincottRaven Publishers, Philadelphia: pp 1839-1849, 1997

17. Brunner MJ, Sussman MS, Greene AS, Kallman $\mathrm{CH}$, and Shoukas AA: Carotid sinus baroreceptor reflex control of respiration. Circ Res 51: 624-636, 1982 